\title{
Transverse Euler Classes of Foliations on Non-atomic Foliation Cycles
}

\author{
Steven HURDER \& Yoshihiko MITSUMATSU
}

\section{Introduction}

The main purpose of this article is to give a geometric proof of the following theorem.

Theorem 1.1 ([4], Theorem 1.10) The average transverse euler class of an almost compact diffuse foliation cycle vanishes.

In [4] this was deduced from the following vanishing theorem.

Theorem 1.2 ([3], Proposition 2, Corollary 3) If a foliation has two foliation cycles and at least one of them is non-atomic, then their homological intersection vanishes. Especially, the self-intersections of nonatomic foliation cycles vanish.

The geometric proof of Theorem 1.1 we give is of independent interest, for it combines a technique of "blowing-up" along foliation cycles with the Hirsch and Thurston vanishing theorem for the Euler class. This was the original method used to discover Theorem 1.1, and has possible further applications. For example, we apply the original idea of Theorem 1.1 to give an alternative proof of Theorem 1.2.

Let $\mathcal{F}$ be a codimension $q C^{2}$-foliation on a closed smooth $n$-manifold $M(n=p+q)$ whose tangent bundle $\tau \mathcal{F}$ and the normal bundle $\nu \mathcal{F}$ are both oriented of dimensions $p$ and $q$ respectively. It is well known (see [8] or [10]) that for orientable foliations, a transverse invariant measure determines a foliation cycle, $C_{\mu}$, and vice versa. A measure is said to be non-atomic if it assigns measure zero to every point. The existence of a non-atomic invariant measure for a dynamical system implies a certain regularity of the orbits in its support.

AMS classification: Primary 57R20, 57R30; Secondary 28D15, 55R25

The first author was supported in part by NSF Grant DMS 91-03297 and a Sloan Foundation Fellowship. The authors are grateful to the University of Colorado at Boulder for its visitor financial support of both authors, which made this collaboration possible.

This paper is in final form and no version of it will be submitted for publication elsewhere. 
A similar principle holds for foliations: a transverse measure is nonatomic if it assigns measure zero to every compact leaf. The existence of an invariant, non-atomic transverse measure $\mu$ reflects on the geometry of leaves in its support and their asymptotic distribution in the transversal space. It also has consequences for the cohomological invariants associated to the foliation, restricted to the support of the measure. In this article, as stated above, we investigate the the cap products $\mathcal{E}(\nu \mathcal{F}, \mu)$ of the normal bundle with foliation cycles, which are called average transverse euler classes. For simplicity, we only deal with non-negative measures. Every (co-)homology has the coefficients in the real numbers $\mathbf{R}$ if not specified.

Remarks 1) The Pontrjagin classes of the normal bundle $\nu \mathcal{F}$ are trivial on the foliation cycles, because of the Chern-Weil theory and the existence of a Bott connection (see [1]).

2) The only known examples of nontrivial cap product $\mathcal{E}(\nu \mathcal{F}, \mu)$ are given by compact leaves, i.e., transverse atomic measures (see [4] or [5]). Therefore it is conjectured that:

Conjecture If a transverse invariant measure $\mu$ is non-atomic, the average euler class $\mathcal{E}(\nu \mathcal{F}, \mu)$ vanishes.

Theorem 1.1 gives a partial answer to this conjecture. A foliation cycle $C_{\mu}$ is said to be almost compact if it satisfies the following conditions on its support.

- There exists a $p$-dimensional closed submanifold $K$ whose tubular neighborhood $N$ is identified with its normal $D^{q}$-bundle, and the support $\operatorname{supp}(\mu)$ is contained in the interior of $N$.

- Each fiber disk $D^{q}$ of $N \rightarrow K$ is transverse to $\mathcal{F}$.

Such a measure $\mu$ is said to be diffuse if the intersection of the support of $\mu$ with a typical fiber of $N$ has cardinality $\#\left\{D^{q} \cap \operatorname{supp}(\mu)\right\}>1$. The holonomy invariance of $\mu$ allows us to normalize $\mu$ so that $\mu\left(D^{q}\right)=1$ for all fibers. Then the corresponding foliation cycle $C_{\mu}$ represents the fundamental class $[K]$ of $K$ in the homology. Therefore, Theorem 1.1 is equivalent to the next result which can be considered as a disk bundle version of Hirsch and Thurston's vanishing theorem of the euler class (see $3.1,3.3$, and [2]).

Theorem 1.3 Let $\mathcal{F}$ be a codimension $q$ foliation on a $D^{q}$-bundle $\xi$ which is transverse to each fibre of $\xi$ and $\mu$ be a transverse invariant measure for $\mathcal{F}$ whose support is contained in the interior of the total space. If $\#\left\{D^{q} \cap \operatorname{supp}(\mu)\right\}>1$, then $e(\xi)=0$. 


\section{Sullivan's Theorem}

In this section we review the following theorem of Sullivan, whose idea is similar to that of the reduction of the proof of Theorem 1.1 to the non-atomic case.

Theorem 2.1 (Sullivan, [9]) Let $\zeta$ be an $R^{q}$-vector bundle whose structural group reduces to $S L(q, \mathbf{Z})$. Then its euler class $e(\zeta)$ vanishes.

To prove this theorem, use the reduction of the holonomy group to form an associated $T^{q}$-bundle $\rho$. Each fibre is identified with $\mathbf{R}^{q} / \mathbf{Z}^{q}$ up to the action of $S L(q, \mathbf{Z})$. Then, this bundle has a multisection $S$ of multiplicity $2^{q}$ which corresponds to half-integer lattice points. It is obvious that $S$ splits into the original 0 -section $S_{0}$ and the remainder $S_{1}$. The important points are that

1) In rational homology, $\left[S_{1}\right]=\left(2^{q}-1\right)\left[S_{0}\right]$.

2) The homological self-intersection $\left[S_{0}\right]^{2}$ vanishes, because $S_{0}$ and $S_{1}$ are actually disjoint, so that we have $\left[S_{0}\right]^{2}=\left(2^{q}-1\right)^{-1}\left[S_{0}\right] \cdot\left[S_{1}\right]=0$.

In this case, 1) follows from that the covering map $D: \rho \rightarrow \rho$ of degree $2^{q}$ given by the fiberwise multiplication by 2 induces an isomorphism on Q-homology and that $\rho_{*}(S)=2^{q} S_{0}$.

Arguments similar to 1) and 2) apply to prove the following results (see also [7]).

Proposition 2.2 If an oriented $2 k$-sphere bundle $\eta$ has a multisection $S$ of degree $>1$, then $\langle e(\tau \eta),[S]\rangle=0$, where $\pi \eta$ denotes the tangent bundle along the fibres.

Proposition 2.3 If an oriented $2 k$-disk bundle $\xi$ has a multisection $C$ of degree $>1$, we have $e(\xi)=0$ over $\mathbf{Q}$.

The Reduction Process: In general, a transverse invariant measure $\mu$ for a foliation $\mathcal{F}$ splits into the non-atomic part $\mu^{c}$ and the atomic part $\mu^{a}$ (i.e., $\mu=\mu^{c}+\mu^{a}$ ), either of which is again an invariant transverse measure for $\mathcal{F}$. In the case of Theorem 1.3, both $C_{\mu^{a}}$ and $C_{\mu^{c}}$ define the same homology class as $C_{\mu}$ up to a scalar multiple, if they are nontrivial. In the case of $\mu^{a}=0$, we prove the vanishing $\mathcal{E}\left(\nu \mathcal{F}, \mu^{c}\right)=0$ (which implies $e(\xi)=0)$ in Section 4. In the case that $\mu^{c}=0$ and $\mu^{a} \neq 0$, the finiteness of $\mu^{a}$ implies that $\operatorname{supp}\left(\mu^{a}\right)$ contains a multisection. Then, Proposition 2.3 implies $e(\xi)=0$. 


\section{The Hirsch-Thurston Theorem, and Blowing- Up of Foliated Manifolds}

In this section, we review two fundamental ideas: the Hirsch-Thurston Theorem in 3.1, and the blowing-up of foliated manifolds in 3.2. The proofs of Theorem 1.1 and Theorem 1.2 do not strictly require the HirschThurston theorem, but we recall it as it gives a basic idea for the key strategy (cf. [4]).

\subsection{The Hirsch-Thurston Theorem}

Let $\eta$ be a foliated bundle $p: E \rightarrow B$ which has a compactly supported, invariant transverse measure $\mu$ and corresponding foliation cycle $C_{\mu}$.

Theorem 3.1 ([2]) The homomorphism $p_{*}: H_{*}(E ; \mathbf{R}) \rightarrow H_{*}(B ; \mathbf{R})$ surjects. Consequently, in the case that $\eta$ is a foliated sphere bundle, $\eta$ has a trivial euler class.

If $\eta$ has a section $s$, the theorem is easy, because ' $s \circ p=i d_{X}$ ' implies $s_{*}$ and $s^{*}$ are (co-)homological transfer maps. In general, foliation cycles give rise to transfer maps. Here, we assume that the fibre $F$ of $\eta$ has $\mu$-measure 1. Then the cohomological transfer map $\sigma$ is constructed on the de Rham complexes as

$$
\sigma(\omega)_{b}=\int_{F_{b} \ni y}\left(p_{* y} \circ l_{y}^{*}\right)(\omega) d \mu(y) \quad \text { for } \omega \in \Omega(E) \text { and } b \in B
$$

where $l_{y}$ denotes the restriction to the leaf through a point $y \in F_{b}$. The homological transfer $\rho$ is defined to be the dual to $\sigma$.

For the case of a sphere bundle, the Euler class can be defined as the transgression of the fiber fundamental class. Hence the Euler class lifted to $H^{*}(E ; \mathbf{R})$ is canonically trivial, and $p^{*}: H^{*}(B ; \mathbf{R}) \rightarrow H^{*}(E ; \mathbf{R})$ injects implies the Euler class is zero in $H^{*}(B ; \mathbf{R})$.

Remarks 1) As is seen from the above, the existence of some geometric objects which give rise to a transfer is the essential point, such as a multisection of finite degree, a transverse foliation with an invariant measure, or measured lamination. Such situations may happen even if the bundle does not admit a flat structure.

2) If we use the singular homology theory with coefficients in compact support Radon measures (see [6]), we obtain a homological transfer in more direct way. 


\subsection{Blowing-up}

The concept of the blowing-up of a codimension $q C^{r}$-foliated manifold $(M, \mathcal{F})$ along a closed saturated $C^{r}$-submanifold $X$ was introduced in [4]. We use the blowing-up procedure that replaces the submanifold $X$ with its normal sphere bundle, rather than with its normal projective space bundle, to obtain:

Proposition $3.2\left([4]\right.$, Section 6) There exist a codimension $q C^{r-1}$ foliated manifold $(\tilde{M}, \tilde{\mathcal{F}})$, a closed saturated $C^{r-1}$-submanifold $S X \subset \tilde{M}$, and a $C^{r-1}$-blowing-down map $\beta: \tilde{M} \rightarrow M$ satisfying:

1) $\beta$ is a leafwise $C^{r-1}$-diffeomorphism.

2) $\beta(S X)=X$ and $\left.\beta\right|_{S X}: S X \rightarrow X$ is the normal sphere bundle projection.

3) $\left.\beta\right|_{\tilde{M} \backslash S X}: \tilde{M} \backslash S X \rightarrow M$ is a foliation preserving $C^{r}$-diffeomorphism.

If $X$ is a null set of a transverse invariant measure $\mu$ of $\mathcal{F}, \mu$ gives rise to a transverse invariant measure $\tilde{\mu}$ of $\tilde{\mathcal{F}}$, with respect to which $S X$ is also a null set.

Proposition 3.3 The blowing-down map $\beta$ pushes the corresponding foliation cycle $C_{\tilde{\mu}}$ down to $C_{\mu}$, i.e., $\beta_{\#} C_{\tilde{\mu}}=C_{\mu}$.

\subsection{Vanishing of euler class}

As an application of the above two propositions, we obtain the following vanishing criterion for the average Euler class:

Proposition 3.4 Let $\mathcal{F}$ be a (not necessarily complete) codimension $q$ foliation on the total space $E$ of an oriented $D^{s}$-bundle $p: E \rightarrow B$, for $s \leq q$, which admits a transverse invariant measure $\mu$ whose support supp $(\mu)$ is contained in the interior of $E$. If there exists a cross section $X$ which is a saturated submanifold and a null set with respect to $\mu$, then the cap product $e(\xi) \cap p_{*}\left(\left[C_{\mu}\right]\right)=0$, or equivalently $p^{*}(e(\zeta)) \cap\left[C_{\mu}\right]$ vanishes.

The proof is now easy. Use the blowing-up procedure along $X$ to obtain $\beta:\left(\tilde{E}, \tilde{\mathcal{F}}, C_{\tilde{\mu}}\right) \rightarrow\left(E, \mathcal{F}, C_{\mu}\right)$. Then, $p \circ \beta: \tilde{E} \rightarrow B$ is an associated annulus bundle, which has the same homotopy type as the sphere a bundle of $E$. Hence, the Euler class of the base is the transgression of the fiber class of $\tilde{E}$, and the Euler class lifts to a canonically trivial class in $H^{*}(\tilde{E} ; \mathbf{R})$. Therefore,

$$
\begin{aligned}
0 & =(p \circ \beta)_{*}\left((p \circ \beta)^{*} e(p) \cap\left[C_{\tilde{\mu}}\right]\right) \\
& =p_{*}\left(p^{*}(e(p)) \cap\left[C_{\mu}\right]\right)=e(p) \cap p_{*}\left(\left[C_{\mu}\right]\right)
\end{aligned}
$$

is obtained. Note that the blowing-up procedure compactifies the complement of the cross-section $X$, so that we can form the current $C_{\mu}$ from the measure $\mu$. 


\section{Proof of Theorem 1.1}

We now give a geometric proof of Theorem 1.1 using the methods reviewed in previous sections. We follow the notation and the assumption of Theorem 1.1. As stated before, we may assume that the transverse invariant measure $\mu$ is non-atomic. We make the following choices:

- a Riemannian metric on $M$

- a closed tubular disk bundle neighborhood $N$ of $K$

- a smaller closed tubular neighborhood $U$ of $K$ such that $\operatorname{supp}(\mu) \subset U \subset N$

satisfying conditions to be described next (see the figure below). Let $E_{N}$ be the unit disk subbundle of the restriction $\left.\nu \mathcal{F}\right|_{N}$ of $\nu \mathcal{F}$ to $N$ and $\left.E_{U} \subset \nu \mathcal{F}\right|_{U}$ be the unit disk subbundle of the restriction to $U$. We assume that the exponential map $\varepsilon: E_{N} \rightarrow M$ is a submersion, $\left.\varepsilon\right|_{L}:\left.E_{N}\right|_{L} \rightarrow M$ is a diffeomorphism for each leaf $L$ of $\left.\mathcal{F}\right|_{N}$, and the induced foliation $\mathcal{F}_{\varepsilon}=\varepsilon^{-1} \mathcal{F}$ on $E_{N}$ is transverse to the bundle fibration $\pi: E_{N} \rightarrow N$. Furthermore we may assume that the inverse image $\varepsilon^{-1}(m)$ of each point $m \in U$ intersects with $\pi^{-1}(U)$ within the interior of $\varepsilon^{-1}(m)$.

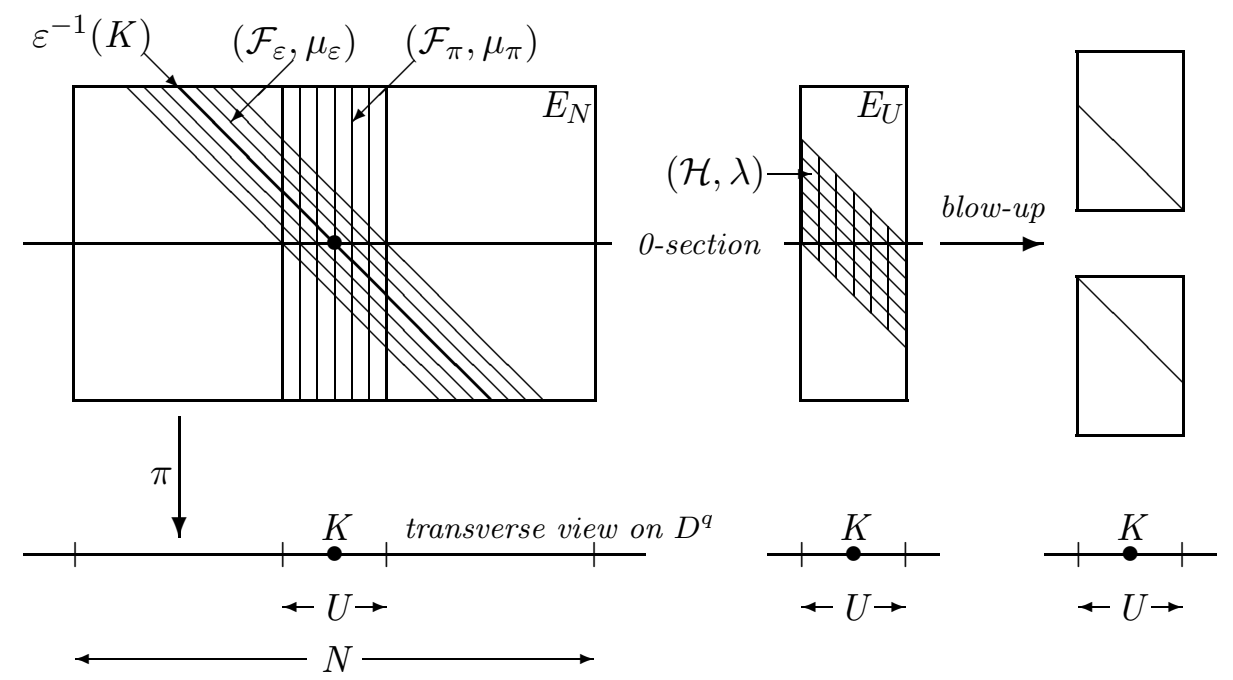

Then, we have two foliations $\mathcal{F}_{\varepsilon}=\varepsilon^{-1} \mathcal{F}$ and $\mathcal{F}_{\pi}=\pi^{-1} \mathcal{F}$ on $E_{N}$, which also have the induced transverse invariant measures $\mu_{\varepsilon}$ and $\mu_{\pi}$. Observe 
that both foliations are not complete, i.e., the boundary $\partial H_{1}$ is neither tangent to $\mathcal{F}_{\varepsilon}$ nor to $\mathcal{F}_{\pi}$. The following two lemmas are easy consequences of our construction.

Lemma 4.1 There exists a codimension $2 q$ foliation $\mathcal{H}=\mathcal{F}_{\varepsilon} \cap \mathcal{F}_{\pi}$ on $E_{N}$ which admits a transverse invariant measure $\lambda=\mu_{\varepsilon} \times \mu_{\pi}$. The foliation $\mathcal{H}$ may fail to be complete, but $\left.\mathcal{H}\right|_{\text {supp }(\lambda)}$ is complete in the leaf direction as $\operatorname{supp}(\lambda) \subset E_{N}$ and $\operatorname{supp}(\lambda) \subset E_{U}$.

Actually, we can consider that $\operatorname{supp}(\lambda)$ is contained in the fibre product $\varepsilon^{-1} U \oplus \pi^{-1} U$ over $K$. Therefore, for each $m \in K$, we have the product coordinate as $D_{m}^{q} \times D_{m}^{q}$ around $\pi^{-1} D_{m}^{q} \cap \operatorname{supp}(\lambda)$, where $D_{m}^{q}$ is the fibre over $m$ of the normal bundle projection : $U \rightarrow K$.

Lemma 4.2 1) With respect to the above product coordinate, the 0-section $S_{0}$ is the diagonal subset $\Delta$.

2) The 0-section $S_{0}$ is a codimensionq closed submanifold of $\left(E_{U}, \mathcal{H}\right)$.

3) The corresponding foliation cycle $C_{\lambda}$ to $\lambda$ is pushed down to $C_{\mu}$ as a current, i.e., $\pi_{\#}\left(C_{\lambda}\right)=C_{\mu}$.

This lemma and a general lemma on measures below imply the next proposition, which is the key observation.

Proposition 4.3 The 0-section $S_{0}$ has measure zero with respect to the transverse measure $\lambda$.

Lemma 4.4 If a Borel measurable space $Y$ has two probability measures $\mu_{1}$ and $\mu_{2}$ and at least one of them has no atoms, then the diagonal subset $\Delta \subset Y \times Y$ has measure zero with respect to the product measure $\lambda=\mu_{1} \times \mu_{2}$. Especially, $\Delta$ is a null set with respect to $\lambda=\mu \times \mu$ if $\mu$ is non-atomic.

Now, using the observation in 3.3, the proof of Theorem 1.1 is completed as follows. Lemma $4.2-3$ ) implies that

$$
\mathcal{E}(\nu \mathcal{F}, \mu)=e(\nu \mathcal{F}) \cap\left[C_{\mu}\right]=\pi_{*}\left(\pi^{*} e(\nu \mathcal{F}) \cap\left[C_{\lambda}\right]\right) .
$$

On the other hand, Proposition 3.4 and Proposition 4.3 give us

$$
\pi^{*} e(\nu \mathcal{F}) \cap\left[C_{\lambda}\right]=0
$$

to complete the proof.

Remark In [4], the proof of Theorem 1.1, in the notation of this section, was obtained from Theorem 1.2 as follows:

$$
\mathcal{E}(\nu \mathcal{F}, \mu)=e(\nu \mathcal{F}) \cap\left[C_{\mu}\right]=e(\nu \mathcal{F}) \cap[K]=[K] \cdot[K]=\left[C_{\mu}\right] \cdot\left[C_{\mu}\right]=0 .
$$




\section{$5 \quad$ Vanishing of Intersections}

As promised in the introduction, we give in this section an alternative proof of Theorem 1.2 using the ideas of the previous sections. Let $\mu_{1}$ and $\mu_{2}$ be transverse invariant measures of $(M, \mathcal{F})$ and assume that one of them has no atoms. To make the description concise, we recall some of the constructions in [7].

First, fix a Riemannian metric on $M$ and let $E$ be the disk bundle of radius 1 of $\nu \mathcal{F}$. After rescaling, we may assume that the restriction of the exponential map $\varepsilon$ to each leaf $L$

$$
\left.\varepsilon\right|_{L}:\left.E\right|_{L} \longrightarrow M
$$

is an immersion. There exists a closed $q$-form $\Phi$ supported on the interior of $E$, so called the Thom form of $E$.

Lemma $5.1([3,6,7])$ The closed $q$-form on $M$

$$
\Phi_{\mu}=\left.\int_{M / \mathcal{F} \ni L}\left(\left.\varepsilon\right|_{L}\right)_{*} \Phi\right|_{\left.E\right|_{L}} d \mu(L)
$$

represents the Poincaré dual cohomology class to the foliation cycle $\left[C_{\mu}\right]$ in $H^{q}(M)$ and thus we obtain

$$
\left[C_{\mu_{1}}\right] \cdot\left[C_{\mu_{2}}\right]=\Phi_{\mu_{1}} \cap\left[C_{\mu_{2}}\right] \in H_{p-q}(M) .
$$

Remarks 1) In $[3,6,7]$, the vanishing of the cohomology class $\left[\Phi_{\mu}\right]^{2}$, which is the Poincaré dual to the self-intersection $\left[C_{\mu}\right] \cdot\left[C_{\mu}\right]$, is discussed. 2) The integral formula (1) is justified by using a partition-of-unity to reduce the question to a local calculation (see [10,3]).

3 ) Let $M / \mathcal{F}$ denote the "local transverse spaces" to $\mathcal{F}$ and $\mu$ the quotient measure there. The foliation cycle $C_{\mu}$ associated to a transverse invariant measure $\mu$ is described in this notation as

$$
C_{\mu}=\int_{M / \mathcal{F} \ni L}\left(\int_{L}\right) d \mu(L)
$$

where " $\int_{L}$ " denotes the current defined by the integration on $L$. We use this kind of expression below.

Now we make a construction which is similar to that of the previous section. Take two foliations $\mathcal{F}_{\varepsilon}=\varepsilon^{-1} \mathcal{F}$ and $\mathcal{F}_{\pi}=\pi^{-1} \mathcal{F}$ on $E$. Here again, they are transverse to each other and define the third foliation $\mathcal{H}=\mathcal{F}_{\varepsilon} \cap \mathcal{F}_{\pi}$. Two transverse invariant measures $\mu_{1}$ and $\mu_{2}$ are also pulled back to $(E, \mathcal{H})$ by $\varepsilon$ and $\pi$ as $\mu_{\varepsilon}=\mu_{1_{\varepsilon}}$ and $\mu_{\pi}=\mu_{2 \pi}$, and they define the transverse invariant measure $\lambda=\mu_{\varepsilon} \times \mu_{\pi}$ of $\mathcal{H}$. 


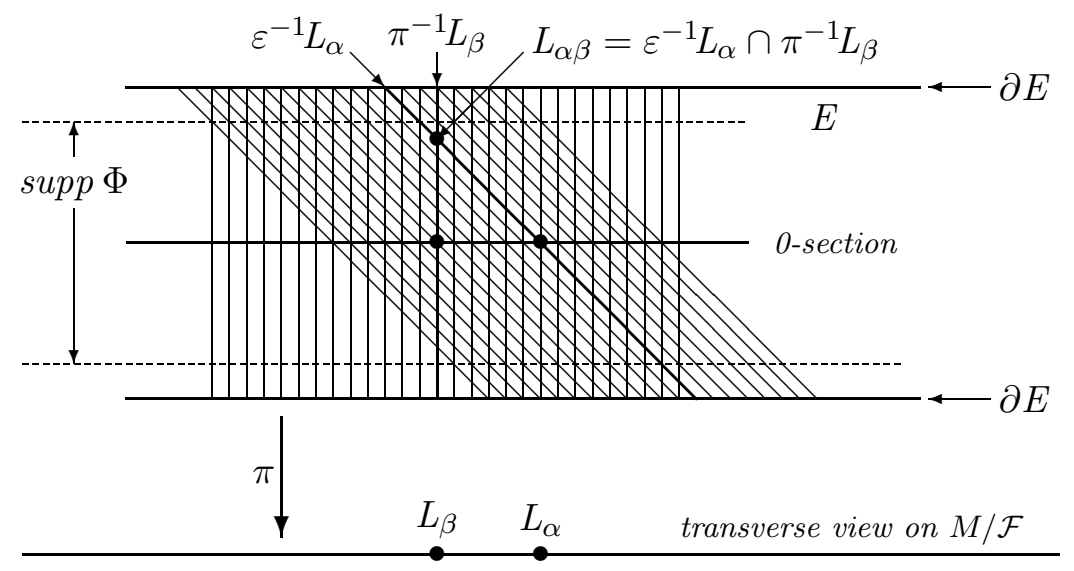

In this case, not only is $\mathcal{H}$ incomplete, but so is $\lambda$. Thus $C_{\mu}$ fails to be a closed current. However, the 0 -section $X$ is a closed saturated submanifold and a null set with respect to $\lambda$.

Lemma 5.2 The foliation cycle current $C_{\lambda}$ is relatively closed in $(E, \partial E)$ and thus defines a homology class in $H_{p}(E, \partial E)$.

The following observation is crucial.

Proposition 5.3 As a current,

$$
\Phi_{\mu_{2}} \cap C_{\mu_{1}}=\varepsilon_{\#}\left(\Phi \cap C_{\lambda}\right)
$$

holds. The right hand side defines an absolute homology class by the cap product pairing

$$
\cap: H^{q}(E, \partial E) \otimes H_{p}(E, \partial E) \rightarrow H_{p-q}(E) .
$$

Proof of Proposition 5.3 Locally, leaves of $\mathcal{H}$ are parametrized by the leaves of $\mathcal{F}_{\varepsilon}$ and those of $\mathcal{F}_{\pi}$, and they agree with $\mathcal{F}$ as a local family of leaves. Then, let $L_{\alpha \beta}$ denote the leaf $\varepsilon^{-1}\left(L_{\alpha}\right) \cap \pi^{-1}\left(L_{\beta}\right)$ of $\mathcal{H}$, where $L_{\alpha}$ and $L_{\beta}$ are nearby leaves of $\mathcal{F}$. Then the following computation shows Proposition 5.3. For any $(p-q)$-form $\omega$ on $M$,

$$
\begin{aligned}
& \left\langle\omega,\left(\Phi_{\mu_{2}} \cap C_{\mu_{1}}\right)\right\rangle=\left\langle\omega \wedge \Phi_{\mu_{2}}, C_{\mu_{1}}\right\rangle \\
= & \left\langle\left.\int_{M / \mathcal{F} \ni L_{\beta}} \omega \wedge\left(\left.\varepsilon\right|_{L_{\beta}}\right)_{*} \Phi\right|_{\left.E\right|_{L_{\beta}}} d \mu_{2}\left(L_{\beta}\right), \int_{M / \mathcal{F} \ni L_{\alpha}}\left(\int_{L_{\alpha}}\right) d \mu_{1}\left(L_{\alpha}\right)\right\rangle \\
= & \int_{M / \mathcal{F} \ni L_{\beta}} \int_{M / \mathcal{F} \ni L_{\alpha}}\left\{\left.\int_{L_{\alpha}} \omega \wedge\left(\left.\varepsilon\right|_{L_{\beta}}\right)_{*} \Phi\right|_{\left.E\right|_{L_{\beta}}}\right\} d \mu_{1}\left(L_{\alpha}\right) d \mu_{2}\left(L_{\beta}\right)
\end{aligned}
$$




$$
\begin{aligned}
& =\int_{(M / \mathcal{F})^{2} \ni\left(L_{\alpha}, L_{\beta}\right)}\left\{\int_{\left(\left.\varepsilon\right|_{L_{\beta}}\right)^{-1}\left(L_{\alpha}\right)}\left(\left.\varepsilon\right|_{L_{\beta}}\right)^{*} \omega \wedge \Phi\right\} d \mu_{1} \times d \mu_{2}\left(L_{\alpha}, L_{\beta}\right) \\
& =\int_{\mathcal{H} \ni L_{\alpha \beta}}\left\{\int_{L_{\alpha \beta}} \varepsilon^{*} \omega \wedge \Phi\right\} d \lambda\left(L_{\alpha \beta}\right) \\
& =\left\langle\varepsilon^{*} \omega, \Phi \cap C_{\lambda}\right\rangle=\left\langle\omega, \varepsilon_{\#}\left(\Phi \cap C_{\lambda}\right)\right\rangle .
\end{aligned}
$$

Then, the proof of Theorem 1.2 goes along a similar way. Apply the blowing-up procedure to $\left(E, \mathcal{H}, C_{\lambda}\right)$ along the 0 -section $X$ to obtain $\left(\tilde{E}, \tilde{\mathcal{H}}, C_{\tilde{\lambda}}\right)$ and the new boundary $S X$. The old boundary is again denoted by $\partial E$. The blowing-down map $\beta$ induces a homology homomorphism $\beta_{*}: H_{*}(\tilde{E}, \partial E) \rightarrow H_{*}(E, \partial E)$ and pushes the relative cycle $C_{\tilde{\lambda}}$ down to $C_{\lambda}$. As $\partial E$ is a deformation retract of $\tilde{E}$, it is clear that

$$
H_{*}(\tilde{E}, \partial E)=H^{*}(\tilde{E}, \partial E)=0,
$$

and of course $\left[C_{\lambda}\right]=\left[C_{\tilde{\lambda}}\right]=0$. 


\section{References}

[1] R.Botт Lectures on Characteristic Classes and Foliations. Springer L.N.M. 279 (1972).

[2] M.Hirsch and W.Thurston Foliated bundles, invariant measures, and flat bundles. Annals of Math. 102 (1975), 369-390.

[3] S.Hurder and Y.Mitsumatsu The intersection product of transverse invariant measures. Indiana Univ. Math. J. 40 (1991), 11691183.

[4] S.HuRder and Y.Mitsumatsu The transverse euler class for amenable foliations. preprint, (1990).

[5] J.Milnor On the existence of a connection with curvature zero. Comment. Math. Helv. 32 (1958), 215-223.

[6] Y.Mitsumatsu Self-intersections and Transverse Euler Numbers of Foliation cycles. PhD thesis, University of Tokyo,(1985).

[7] Y.Mitsumatsu On the self-intersections of foliation cycles. Trans. A. M. S. 334 (1993), 851-860.

[8] D.Ruelle and D.Sullivan Currents, flows, and diffeomorphisms. Topology 14 (1975), 319-327.

[9] D.Sullivan La classe d'Euler réele d'un fibré vectoriel à groupe structural $S L_{n}(\mathbf{Z})$ est nulle. C. R. Acad. Sc. Paris 281 (1975), 17-18.

[10] D.Sullivan Cycles for the dynamical study of foliated manifolds and complex manifolds. Invent. Math. 36 (1976), 225-255.

Steven HURDER

Department of Mathematics (M/C 249)

University of Illinois at Chicago

CHICAGO, IL 60680-4348

E-mail: hurder@boss.math.uic.edu
Yoshihiko Mitsumatsu

Department of Mathematics Chuo University

1-13-27 Kasuga, Bunkyo-ku

TOKYO 112, JAPAN

E-mail: yoshi@indsys.chuo-u.ac.jp yoshi@math.chuo-u.ac.jp 